\title{
Test-beam activities and results for the ATLAS ITk pixel detector
}

\author{
T. Bisanz ${ }^{a}$ on behalf of the ATLAS collaboration and the ITk system \\ ${ }^{a}$ Georg August Universität Göttingen, \\ II. Physikalisches Institut, \\ Friedrich Hund Platz 1, 37077 Göttingen, \\ Germany \\ E-mail: tobias.bisanz@phys.uni-goettingen.de
}

AbstRact: The Phase-II upgrade of the LHC aims at an increase of the instantaneous luminosity up to about $5 \times 10^{34} \mathrm{~cm}^{-2} \mathrm{~s}^{-1}$. To cope with the resulting challenges the current Inner Detector will be replaced by an all-silicon Inner Tracker (ITk) system. The Pixel Detector will have to deal with occupancies of about $300 \mathrm{hits} / \mathrm{FE} / \mathrm{s}$ as well as a fluence of around $2 \times 10^{16} \mathrm{n}_{\mathrm{eq}} \mathrm{cm}^{-2}$. Various sensor layouts are under development, aiming at providing a high performance, cost effective pixel instrumentation to cover an active area of about $10 \mathrm{~m}^{2}$. These range from thin planar silicon, 3D silicon, to active CMOS sensors.

After extensive characterization of the sensors in the lab, their charge collection properties and hit efficiency are measured in common testbeam campaigns, which provide valuable feedback for improvements of the layout. Testbeam measurements of the final prototypes will be used for the decision of which sensor types will be installed in ITk.

The setups used in the ITk Pixel testbeam campaigns will be presented, including the common track reconstruction and analysis software. Results from the latest measurements will be shown, highlighting some of the developments and challenges for the ITk Pixel sensors.

KeYwords: Particle tracking detectors; Radiation-hard detectors; Large detector systems for particle and astroparticle physics 


\section{Contents}

1 Introduction 1

1.1 High Luminosity LHC 2

1.2 ATLAS ITk Upgrade 2

1.3 ATLAS Pixel Detector 3

2 Testbeam Measurements $\quad 3$

2.1 The Telescope 4

3 Testbeam Data Acquisition 5

4 Testbeam Reconstruction and Analysis $\quad 5$

4.1 Data Conversion 6

4.2 Clustering 6

$\begin{array}{lll}4.3 & \text { Hit Derivation } & 7\end{array}$

4.4 Alignment 7

4.5 Track Fit 8

4.6 Analysis 8

5 Results 9

5.1 Irradiated Thin Silicon Sensors 9

$\begin{array}{llll}5.2 & \text { FE65p2 } & 9\end{array}$

6 Summary and Conclusion $\quad 10$

\section{Introduction}

The Large Hadron Collider (LHC) is a proton-proton collider used to study the fundamental forces and properties of particles, described by the Standard Model (SM) of particle physics. One notable milestone of the experiments located at the LHC was the discovery of the Higgs boson in 2012, predicted by the Brout-Englert-Higgs mechanism [1, 2] within the SM.

This discovery was made simultaneously by the ATLAS [3] and CMS [4] collaborations, each exploiting their general purpose detector which was designed to not only discover the Higgs boson, but also probe the Standard Model and search for physics beyond it.

New physics could help understand unanswered questions in particle physics and related fields as well as improve our understanding of the most basic forces and properties of particles and matter surrounding us. Many models of physics beyond the SM aim at answering questions like the origin of dark matter or why there is an asymmetry between matter and antimatter. 


\subsection{High Luminosity LHC}

The LHC operates at a center-of-mass energy of $\sqrt{s}=13 \mathrm{TeV}$, opening a window to a much larger energy range than previous hadron colliders. The amount of interactions, i.e. the collected physics data, is governed by the luminosity of the accelerator. The current nominal instantaneous luminosity of $\mathcal{L}=1.0 \times 10^{34} \mathrm{~cm}^{-2} \mathrm{~s}^{-1}$ has already been exceeded by LHC operation, which aims to reach $(5-7.5) \times 10^{34} \mathrm{~cm}^{-2} \mathrm{~s}^{-1}$ after the upgrade of the LHC to the High Luminosity LHC (HL-LHC).

While this improves the physics reach by providing much more data and thus allows for searches which require much more statistics, it also poses challenges to the detector systems.

The current ATLAS pixel detector was designed to deal with about $\langle\mu\rangle \approx 40$ proton-proton collisions per event. A complex trigger system issues a read-out of a pixel module at a rate of about $100 \mathrm{kHz}$.

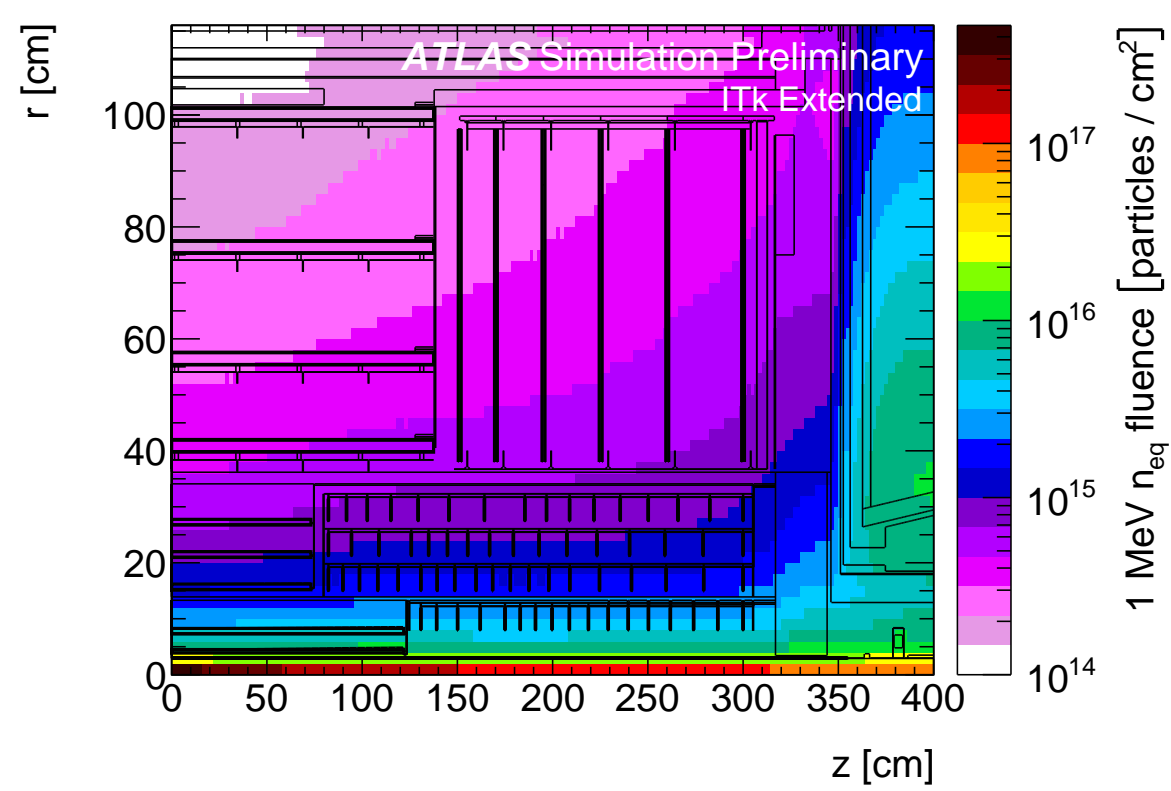

Figure 1. Fluence at the different regions in the ATLAS-inner detector at the HL-LHC.

At HL-LHC conditions, one expects $\langle\mu\rangle \approx 140-200$ [5]. This leads to a higher hit rate as well as higher track density. At the same time, the pixel modules are supposed to be read out at $O(1) \mathrm{MHz}$. While some of these problems can be mitigated by moving to smaller pixel sizes and faster read-out electronics, there is also an increased amount of radiation damage to deal with. At the end of their lifetime, the innermost pixel layers are expected to be irradiated to a total fluence of about $2 \times 10^{16} \mathrm{n}_{\mathrm{eq}} \mathrm{cm}^{-2}$. The overall accumulated fluence at the end of its lifetime for the upgraded detector is shown in Figure 1, where the pixel layers closest to the interaction point are located approximately at $r \approx 4 \mathrm{~cm}$.

\subsection{ATLAS ITk Upgrade}

The innermost detector subsystem of the current ATLAS detector [6] is the Inner Detector. It consists of four layers of pixel modules (originally three, with a fourth one inserted with the 
Insertable B-Layer (IBL) upgrade [7]), four layers of strip detectors and a transition radiation tracker at the outermost section.

This initial pixel detector was designed for a total fluence corresponding to a total of $400 \mathrm{fb}^{-1}$ delivered luminosity, and while the IBL is capable of compensating for efficiency loss due to radiation damage and to exploit the physics content of the the good LHC operation, an entirely new Inner Tracker (ITk) is needed for HL-LHC conditions.

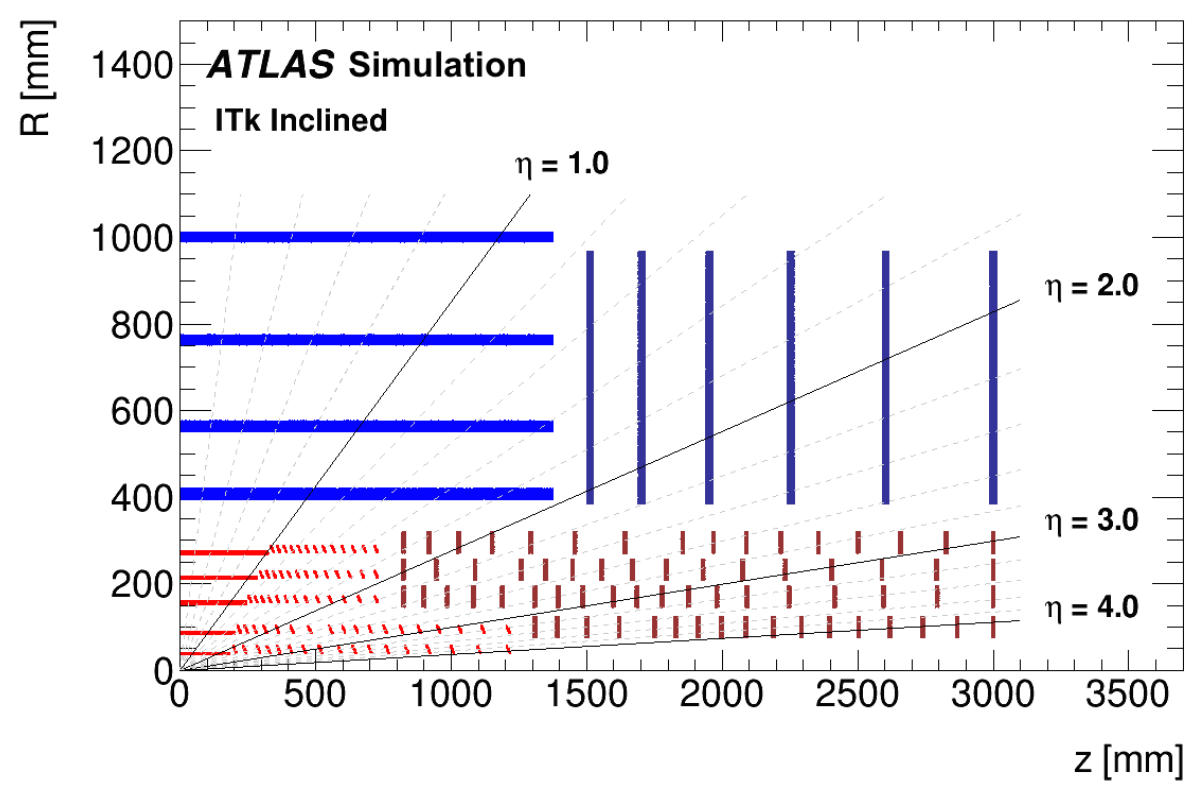

Figure 2. Baseline layout for the ITk upgrade.

The ITk detector will be a pure silicon tracker, i.e. it will be composed of only pixel and strip modules. The baseline layout is shown in Figure 2. It depicts five pixel layers (red) and four strip layers (dark blue) in the barrel section and is immersed in the magnetic field of the existing solenoid magnet (light blue). High angular coverage is achieved with additional six layers of pixel (red) and seven of strip (dark blue) disks [5].

\subsection{ATLAS Pixel Detector}

The ATLAS pixel detector uses hybrid pixel modules. This means, that the sensitive volume where charge is released and collected (the sensor) is physically separated from the amplification and read-out circuitry (the front-end, FE). Sensor and FE are connected via bump bonds, linking each pixel cell on the FE to each pixel implant on the sensor. The most recent FE in the ATLAS detector is the FE-I4B. It uses rectangular pixels of $250 \times 50 \mu \mathrm{m}^{2}$ size and is currently the most used FE in $\mathrm{R} \& \mathrm{D}$ for the ATLAS ITk upgrade.

\section{Testbeam Measurements}

A testbeam facility will provide a controlled beam of charged particles, enabling studying of novel detector components with highly energetic particles. The energy depends on the facility and usually 
ranges from several $\mathrm{GeV}$ to above $100 \mathrm{GeV}$.

Testbeam measurements are an important tool during the investigation of new sensor and FE designs. They accompany measurements in the laboratory by providing an environment which is much closer to the real detector operation than any lab set-up possible.

\subsection{The Telescope}

In order to reconstruct a track of a charged particle, it is necessary to have a set of reference sensors which allow spatially and temporally precise tracking. For the purpose of ATLAS pixel testbeams a copy of the DESY EUDET-like telecope is used. This set-up consists of six Mimosa26 sensors, three upstream and three downstream of the devices under test (DUTs). Such a set-up is depicted in Figure 3. The six Mimosa26 modules with their read-out on top can be seen as well as the DUTs in between the up- and down-stream arm of the telescope.

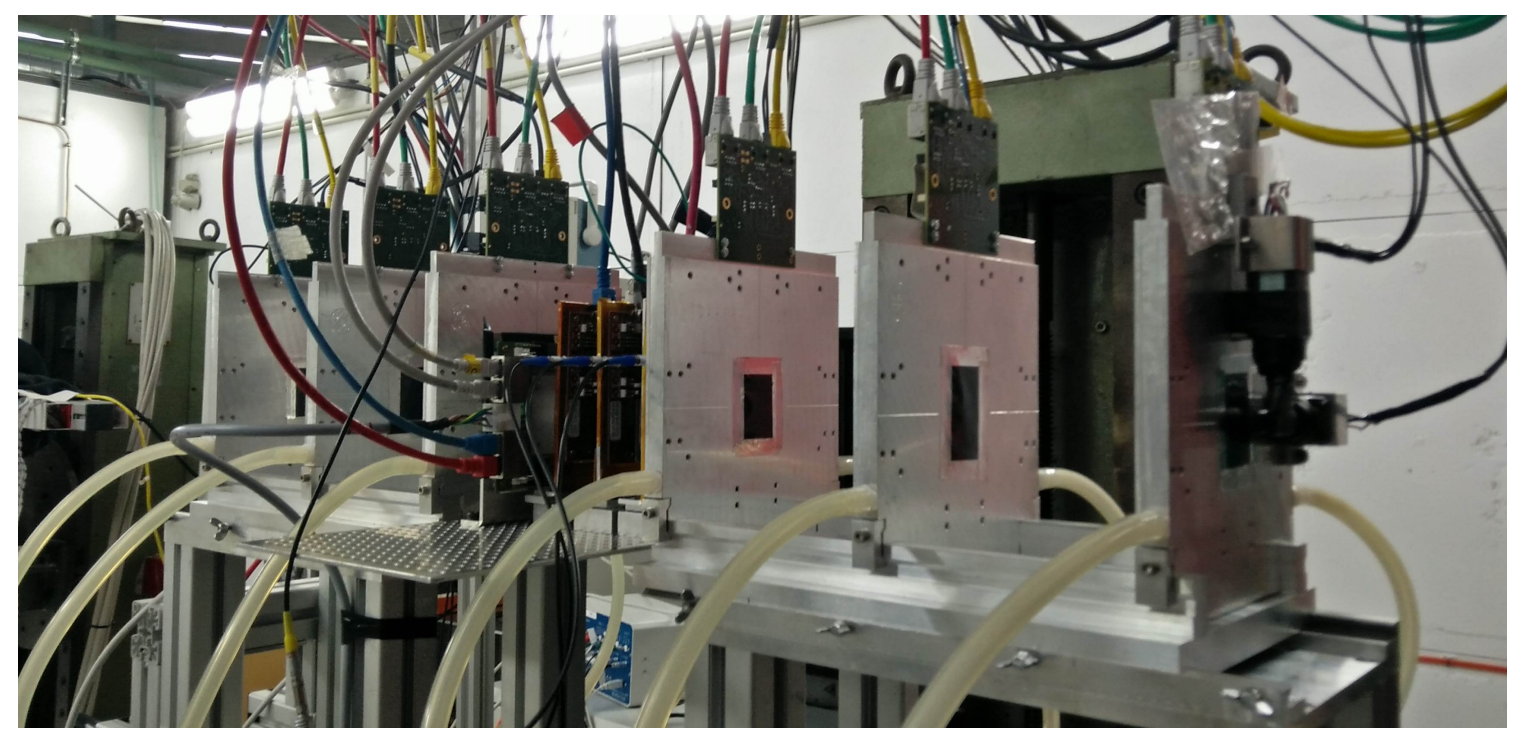

Figure 3. Picture of a typical ATLAS testbeam set-up. Shown are the six telescope planes, with three DUTs between the up- and down-stream arm of the telescope. On the right, a pair of scintillators belonging to the trigger system can be seen.

The Mimosa26 sensors belong to the family of monolithic active pixel sensors, designed by IPHC in Strasbourg [8]. They feature quadratic pixels with $18.4 \mu \mathrm{m}$ pitch in both directions, are thinned down to $50 \mu \mathrm{m}$ and have a rolling shutter read-out, one frame read out every $115.2 \mu \mathrm{s}$.

The intrinsic resolution has been measured to be $(3.24 \pm 0.09) \mu \mathrm{m}$ [9] at typical threshold settings and beam parameters encountered during ATLAS testbeams. This is due to charge sharing between pixels and the measured intrinsic resolution allows to estimate the track resolution at the point of the DUTs, given the spacing and material estimation of the set-up and beam parameters. It has been shown, that the best achievable resolution at the DESY testbeam facility $(5 \mathrm{GeV}$ electron/positron beam $)$ is $(1.83 \pm 0.03) \mu \mathrm{m}$ while $(1.33 \pm 0.03) \mu \mathrm{m}$ can be reached at the CERN SPS testbeam (with $120 \mathrm{GeV}$ pions/kaons), mainly due to the higher energy and thus the lower impact of multiple scattering [9]. 
This resolution not only allows the measurement of global efficiencies of the overall module, but also to perform in-pixel scans, resolving effects of sub-pixel features like bias rails, 3D electrodes or detailed investigation of spatial efficiency distributions at the physical edge of the sensor.

\section{Testbeam Data Acquisition}

In order to correctly associate the data of the telescope and DUTs, the data acquisition (DAQ) systems needs to be synchronised. For this purpose, additional trigger hardware is provided as telescope infrastructure. The Trigger Logic Unit [10] is responsible for receiving triggers from scintillators mounted up- and downstream of the telescope. It offers an interface for user DAQ systems, enabling synchronisation of the telescope and the ATLAS pixel custom DAQ systems by issuing trigger numbers to the various systems attached during a run.

This hardware synchronisation is expanded by the EUDAQ DAQ software framework [11], which provides an easy way to merge the collected data into a single file during data taking. This is accomplished via TCP/IP communication of the multiple DAQ systems, and in addition also allows online data monitoring as well as logging of additional slow control parameters, like leakage currents, temperature etc.

\section{Testbeam Reconstruction and Analysis}

EUTelescope is a testbeam reconstruction framework [12, 13]. It is part of the ilcsoft software collection and based on Marlin, the Modular Analysis \& Reconstruction for the Linear Collider framework. Marlin allows processors to operate on collections. These collections are managed by the back-end I/O framework LCIO (Linear Collider Input/Output). The geometry of the telescope and DUTs is described via an XML document, parsed by GEAR, the Geometry API for Reconstruction.

From this point of view, EUTelescope is a collection of Marlin processors, dedicated to processing of testbeam data. This includes processors to treat noisy pixels, processors to align the telescope and processors to perform track fits. This scheme is shown in Figure 4. Frameworks belonging to ilcsoft are highlighted in blue, EUTelescope in green and external frameworks and tools in red.

The modular design of Marlin makes EUTelescope a very generic and versatile framework. It allows users to easily integrate their DUT geometry and tailor their analyses to custom needs. An example where this flexibility comes into play are the various types of information different DAQ systems provide. While the Mimosa26 only provides binary hit information and no time information, the FE-I4B will provide a 4 bit charge as well as some additional time information. Other sensors might even have multiple time-stamps (e.g. multiple thresholds). All this can be easily incorporated into EUTelescope.

Despite this modularity, most reconstructions follow a very similar scheme, especially within the ATLAS ITk upgrade project, ensuring that results can be compared to each other. 


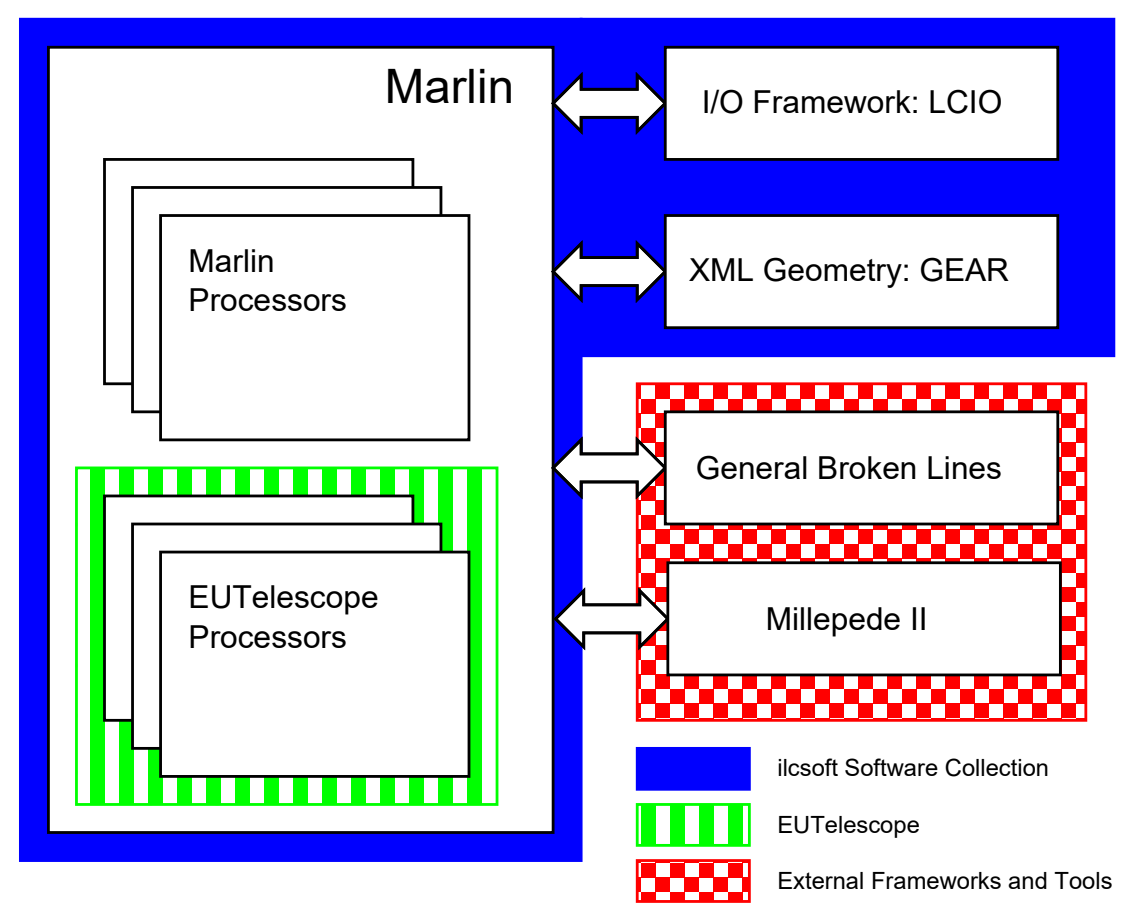

Figure 4. Relation of the different parts of ilcsoft. EUTelescope is shown as a collection of Marlin processors. $\mathrm{I} / \mathrm{O}$ and geometry is handled by LCIO and GEAR via Marlin.

\subsection{Data Conversion}

The ATLAS ITk DAQ systems are fully integrated into EUDAQ, introduced in Section 3. This allows the data conversion to be fully integrated into EUTelescope, i.e. no external tool needs to convert the raw sensor data from the FE-I4B (or novel FEs) into the LCIO format.

A Marlin processer will be called which will execute interpretation of the raw sensor data and turn it into standardised LCIO data. In this step, the firing frequency, defined as the number of events in which a hit was registered divided by the total number of events is determined for each pixel. Pixels above a threshold value (which has to be set by the user, as it depends on the tuning, beam and DAQ parameters) are considered noisy and are stored in an external database.

\subsection{Clustering}

Clustering in EUTelescope is merely a reordering of data in the LCIO collections. Adjacent pixels are grouped together; in which order and what adjacent exactly means can be set by the user. Also noisy pixels can be either removed prior to clustering, or clusters containing noisy pixel can be removed.

The default ATLAS pixel clustering settings define adjacent pixels as pixels which at least touch in the corner, i.e. each pixel has eight neighbours. No temporal cut is applied by default. Noisy pixel treatment is done by masking and removing clusters which contain noisy pixels after clustering.

This leads to a systematic uncertainty which has to be assessed by the user for each analysis, as it depends on the sample. Typically, a fiducial cut is applied during the analysis, removing tracks 
in close proximity to noisy pixels, eliminating (or minimising) any bias due to this.

\subsection{Hit Derivation}

In this step, an actual spatial position on the sensor is derived. This is done by taking all the hit pixels from a cluster and computing the mean position. By default, a charge weighted center of gravity algorithm is used, however also a simple geometric mean is possible.

For strip sensors or two-hit clusters with charge information, it would be possible to perform an $\eta$-correction. However, this is not done by default as the cumulative distribution function needs to be derived from data.

For the FE-I4B it has been shown that the geometric mean (for perpendicular tracks) and the charge weighted mean (for inclined tracks) perform worse only by a small margin, compared to an $\eta$-corrected charge weighted mean [14].

As spatial hit positions are derived in this step, it is possible to obtain a rough estimate of the misalignment- This is in particular of importance, as the actual alignment needs a preliminary track fit, which is not robust against large alignment uncertainties. The prealignment processor assumes that tracks have a perpendicular incidence and thus hits in the global frame should not change in $\mathrm{x}$ - and $\mathrm{y}$-direction, if multiple scattering is neglected. The difference of the hit position on the most upstream plane and all subsequent planes is plotted in a histogram for $\mathrm{x}$ - and y-direction and the GEAR file is corrected for those misalignments. This obviously only works if the coordinate systems of the various planes are not rotated with respect to each other as significant rotations will result in this approach to fail. Furthermore, the angular beam profile must peak at zero for this to not incorrectly bias the prealignment.

However, it must be noted, that the derived prealignment is only used to obtain a better starting point for the subsequent alignment and typically is much coarser (chosen by the bin size of the histogram) than any of the mentioned possible systematic effects.

\subsection{Alignment}

In the alignment step, the prealigned hits are used to perform a track fit. For this purpose, the same processors as the ones for the final track fit are used, but the expected intrinsic resolution of the various detector planes is increased to take the misalignment into account. Depending on the implementation, possibly different track finding algorithms might be used.

ATLAS Pixel by default uses an implementation of a Deterministic Annealing Filter (DAF) [15] fitter [14], a variation of a Kalman filter, to perform the track fit. Multiple scattering is incorporated as process noise and a combination of a forward and backward running filter allow to combine both state predictions to give the best prediction, also on the initial planes.

Residuals from the track fit are then used to obtain the final alignment values. This is done via the Millepede II framework [16, 17]. Millepede II will perform an overall least squares fit, including all the local as well as global parameters. Typically, alignment is done by assuming fixed alignment parameters and estimating corrections to those parameters with a least squares fit of single tracks. This however will only result in a biased alignment. An iterative approach usually tries to minimise this bias, however it is not given that such alignment will converge. 
The overall least squares fit done by Millepede II exploits the mathematical structure of the least square matrix in an overall (i.e. local and global) parameter fit and thus provides unbiased alignment corrections in a non-iterative way.

\subsection{Track Fit}

The final track fit is done after applying the correct alignment derived in the step prior. ATLAS Pixel also uses the implementation of the DAF fitter in EUTelescope. The obtained tracks are then dumped into an external file to be processed by the TBmonII testbeam analysis framework.

In EUTelescope, recently the General-Broken-Line (GBL) algorithm [17, 18] has been implemented, however track-finding is currently limited to a telescope-only set-up.

\subsection{Analysis}

TBmonII is the ATLAS Pixel testbeam analysis framework. It operates on output files produced by EUTelescope. Figure 5 shows the working mechanism of TBmonII. An analysis of testbeam data usually consists of multiple TBmonII analyses. These analyses can be individually configured in a dedicated analysis configuration file, as can be the DUT geometry for every DUT included in the corresponding DUT configuration file. In addition, central configuration parameters, like selection of fiducial regions to accept or reject tracks or track quality criteria, need to be set by the user.

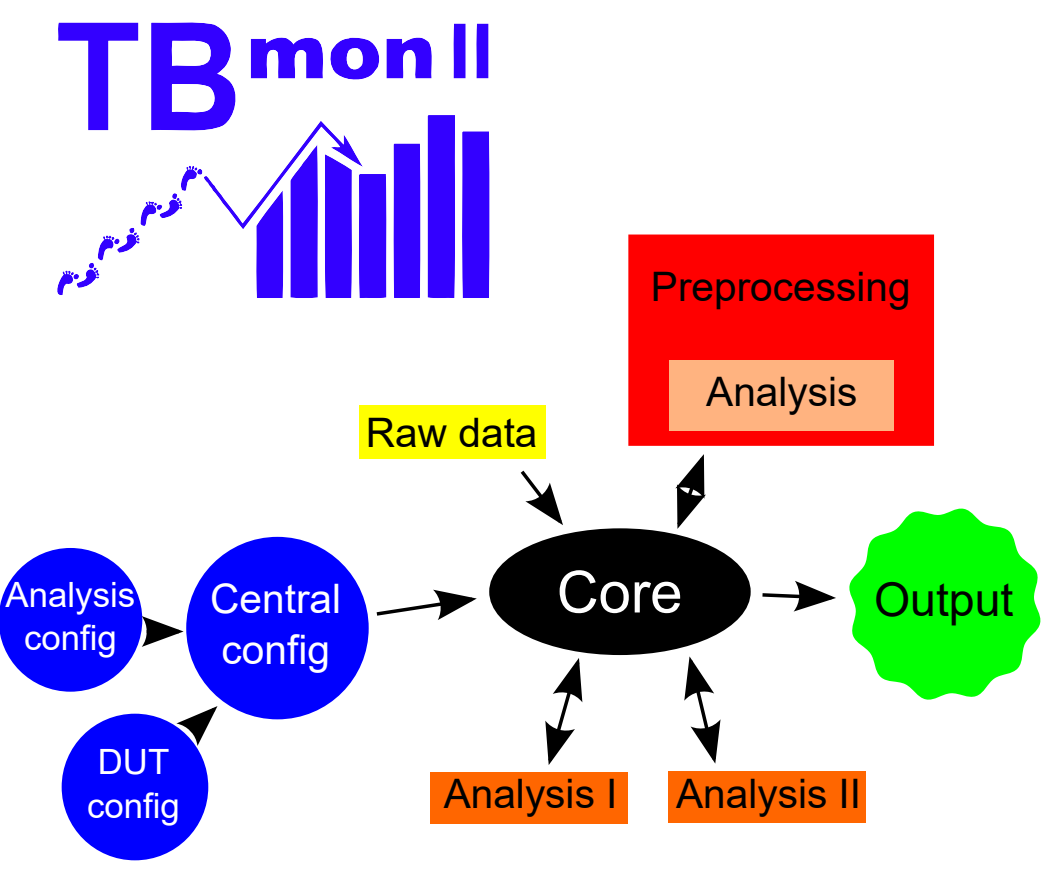

Figure 5. Schematic overview of the working principle of TBmonII.

Once TBmonII is executed, it will select a set of good tracks as defined by selection criteria set by the user, which are used in the subsequent analyses. Furthermore, preprocessing steps like validation of the alignment can be included. 


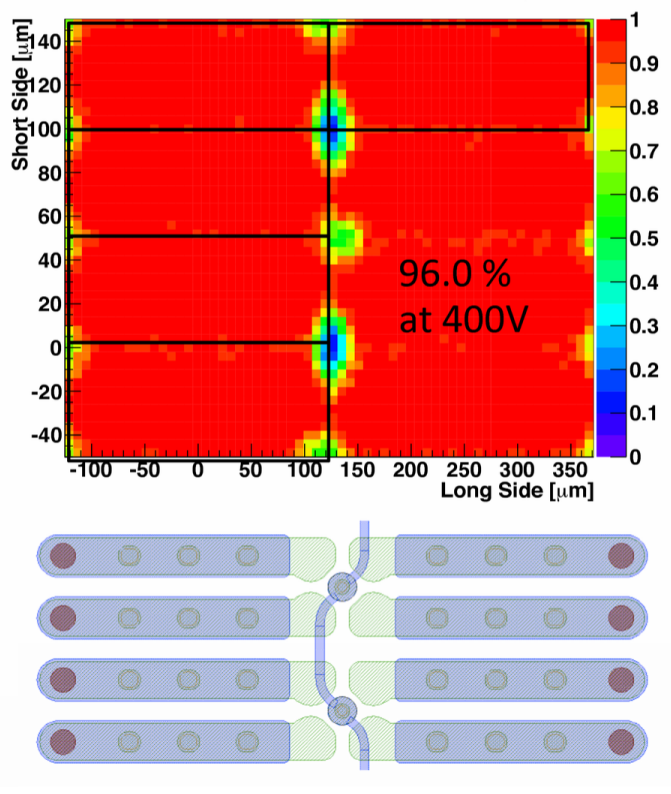

(a) MPG HLL SOI3 layout.

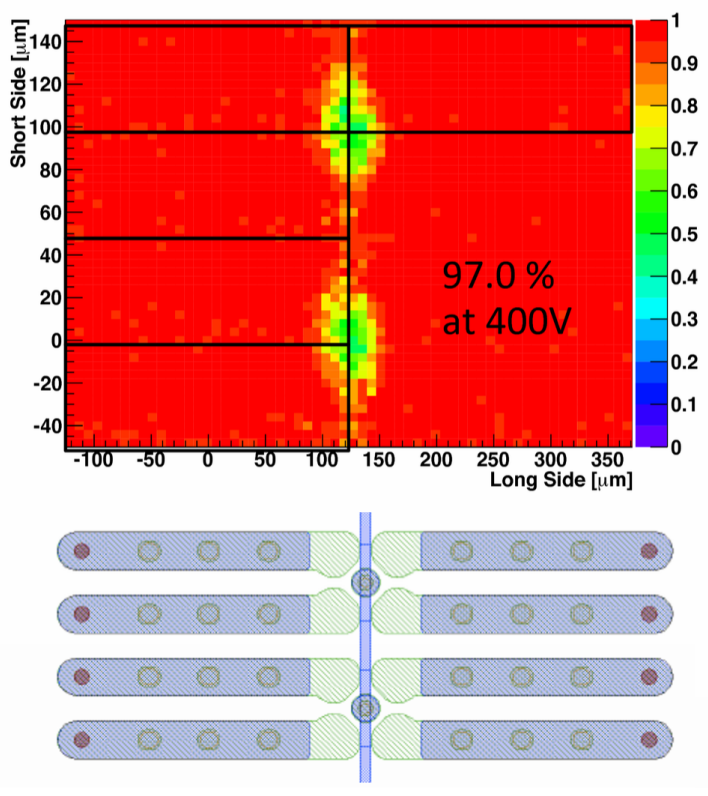

(b) CIS4 layout.

Figure 6. In-pixel efficiency TBmonII analyses for different bias rail implementations after irradiation.

Once preprocessing and track selection is done, the subset of selected tracks is used to perform the analyses. These include global as well as spatially resolved in-pixel efficiency studies, charge sharing properties and edge analysis which all result in standardised output plots.

\section{Results}

\subsection{Irradiated Thin Silicon Sensors}

An example of a TBmonII in-pixel efficiency analysis is shown in Figure 6, where spatial efficiencies for two different implementations of bias rails for $100 \mu \mathrm{m}$ thick planar silicon sensors after irradiation to $5 \times 10^{15} \mathrm{n}_{\mathrm{eq}} \mathrm{cm}^{-2}$ are compared. Both have a punch-through dot with a diameter of $23 \mu \mathrm{m}$, but the MPG HLL SOI3 layout (Figure 6a) features the bias rails on the implants, whereas the CIS4 layout (Figure 6b) has a standard bias rail routing. The obtained sub-pixel resolution during reconstruction allows to spatially resolve the efficiency and can explain the difference in the global efficiency of the two samples.

\section{$5.2 \quad$ FE65p2}

As mentioned in Section 1.1, moving to smaller pixel sizes will help to deal with the increased hit rates and track density. The FE65p2 is a prototype of a read-out chip for pixels of either $100 \times 25$ or $50 \times 50 \mu \mathrm{m}^{2}$ size. First samples have been included in testbeam measurements and recently a full integration into EUDAQ and EUTelescope has been achieved.

The measurement was performed using the DESY EUDET-like telecope, only two upstream planes were used as one was broken. The FE65p2 was placed together with a standard FE-I4 reference module between the two arms of the telescope, enclosed in a cooling box to cool down 


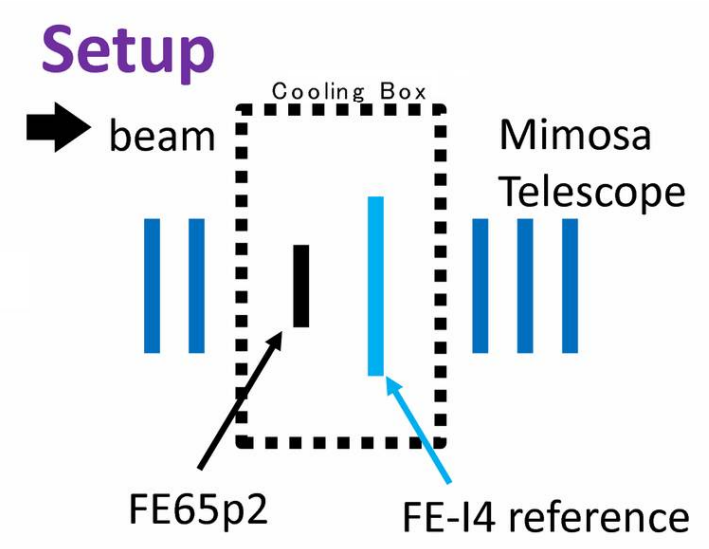

Figure 7. CERN testbeam set-up for the first FE65p2 testbeams with full EUDAQ integration. Only five Mimosa26 planes were used.

the samples up to $-40{ }^{\circ} \mathrm{C}$ to allow measurement of irradiated samples while reducing annealing, sketched in Figure 7.

\section{Summary and Conclusion}

This paper outlines the challenges for the upcoming ATLAS Pixel Detector within the ITk upgrade. The DESY EUDET-like telescopes have been introduced as they serve as the reference instrument for many ATLAS testbeam campaigns. The challenges and possibilities of DAQ system integration into the existing EUDAQ framework has been outlined, especially in regards of synchronisation via the Trigger Logic Unit.

The EUTelescope reconstruction framework is thoroughly used in the ATLAS Pixel community. A detailed summary of the reconstruction steps has been given, placing emphasis on the relevant parts for the ATLAS Pixel R\&D projects.

Obtained tracks are used by TBmonII, the ATLAS Pixel testbeam analysis framework. Multiple analyses produce standardised output plots which allows comparison of results from various testbeam campaigns and groups. An example of such an analysis is the in-pixel efficiency analysis. Results for such an analysis for irradiated thin planar silicon sensors have been shown.

In the near future, a new FE for the ITk upgrade will replace the existing FE-I4B. This FE will need to be implemented into the default testbeam frameworks, work which has already started and is ongoing. Ultimately, this will allow to study novel sensor designs, also with pixel sizes aimed at for the final ITk modules, a notable milestone being the ITk pixel technical design report due by the end of 2017.

\section{Acknowledgments}

The measurements leading to these results have been performed at the Test Beam Facility at DESY Hamburg (Germany), a member of the Helmholtz Association (HGF). 


\section{References}

[1] F. Englert and R. Brout, Broken Symmetry and the Mass of Gauge Vector Mesons, Phys. Rev. Lett. 13 (1964) 321.

[2] Peter W. Higgs, Broken Symmetries and the Masses of Gauge Bosons, Phys. Rev. Lett. 13 (1964) 508.

[3] ATLAS Collaboration, Observation of a new particle in the search for the Standard Model Higgs boson with the ATLAS detector at the LHC, Phys. Lett. B 716 (2012) 1.

[4] CMS Collaboration, Observation of a new boson at a mass of $125 \mathrm{GeV}$ with the CMS experiment at the LHC, Phys. Lett. B 716 (2012) 30.

[5] ATLAS Collaboration, Technical Design Report for the ATLAS Inner Tracker Strip Detector, ATLAS-TDR-025 (2017).

[6] ATLAS Collaboration, The ATLAS Experiment at the CERN Large Hadron Collider, JINST, 3 (2008) S08003.

[7] ATLAS Collaboration, ATLAS Insertable B-Layer Technical Design Report, ATLAS-TDR-19 (2010).

[8] J. Baudot et al., First test results Of MIMOSA-26, a fast CMOS sensor with integrated zero suppression and digitized output, Nuclear Science Symposium Conference Record (NSS/MIC), (2009) IEEE.

[9] H. Jansen et al., Performance of the EUDET-type beam telescopes, EPJ T 3, (2016) 7.

[10] D. Cussans, Description of the JRA1 Trigger Logic Unit (TLU), v0.2c, EUDET-MEMO-2009-04 (2009).

[11] E. Corrin, EUDAQ Software User Manual, EUDET-Memo-2010-01 (2010).

[12] I. Rubinskiy, EUTelescope. Offline track reconstruction and DUT analysis software., EUDET-Memo-2010-12 (2010).

[13] EUTelescope developers team, EUTelescope 1.0: Reconstruction Software for the AIDA Testbeam Telescope, AIDA-NOTE-2015-009 (2015).

[14] H. Gjersdal, Test beam track reconstruction and analysis of ATLAS 3D pixel detectors, Series of dissertations submitted to the Faculty of Mathematics and Natural Sciences, University of Oslo, No. 1573 (2014).

[15] R. Frühwirth and A. Strandlie, Track fitting with ambiguities and noise: A study of elastic tracking and nonlinear filters, Comput. Phys. Commun., 120, (1999) 197.

[16] V. Blobel, Software alignment for Tracking Detectors, NIM A, 566 (2006) 5.

[17] V. Blobel et al., Fast alignment of a complex tracking detector using advanced track models, Comput. Phys. Commun., 183 (2011) 1760.

[18] C. Kleinwort, General broken lines as advanced track fitting method, NIM A, 673 (2012) 107. 